\title{
Evolution and Distribution of Hydrolytic Enzyme Activities during Preharvest Sprouting of Wheat (Triticum aestivum) in the Field
}

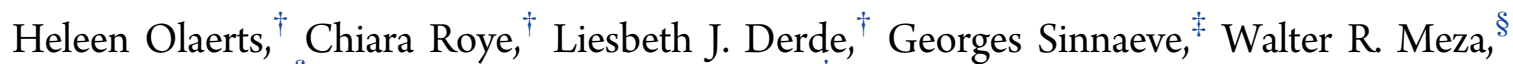 \\ Bernard Bodson, ${ }^{\S}$ and Christophe M. Courtin*, ${ }^{\dagger}$ \\ ${ }^{\dagger}$ Laboratory of Food Chemistry and Biochemistry \& Leuven Food Science and Nutrition Research Centre (LFoRCe), KU Leuven, \\ Kasteelpark Arenberg 20, B-3001 Leuven, Belgium \\ ${ }^{\ddagger}$ Département Valorisation des Productions, Centre Wallon de Recherches Agronomiques (CRA-W), Chaussée de Namur 24, B-5030 \\ Gembloux, Belgium \\ ${ }^{\S}$ Unité de Phytotechnie des Régions Tempérées, Gembloux Agro-Bio Tech (G-ABT), Université de Liége, Passage des Déportés 2, \\ B-5030 Gembloux, Belgium
}

Supporting Information

ABSTRACT: To date, research on preharvest sprouted (PHS) wheat has mostly been conducted on kernels germinated under laboratory conditions, which differ widely from conditions in the field. To obtain detailed knowledge of the evolution of hydrolytic enzyme activities in PHS wheat (Triticum aestivum), a broad collection of samples from three varieties was obtained by harvesting before, at, and after maturity. Delaying harvest time coupled with periods of heavy rainfall caused sprouting in the kernels, observed as a drop in Falling Number and an increase in $\alpha$-amylase activity. The appearance of $\alpha$ - and $\beta$-amylase, peptidase, and endoxylanase activity during field sprouting was independent from each other. Consequently, Falling Number could not be used to predict activity of other hydrolytic enzymes. When differentiating endogenous from kernel-associated microbial enzymes, results showed that $\alpha$ - and $\beta$-amylase and peptidase activity of PHS kernels were predominantly of endogenous origin, whereas endoxylanase activity was largely from microbial origin.

KEYWORDS: Triticum aestivum, field sprouting, Falling Number, $\alpha$-amylases, $\beta$-amylases, peptidases, endoxylanases

\section{INTRODUCTION}

In wheat-based biotechnological processes, variation in flour yield and quality is a major and recurring problem. It is generally known that such variations not only result from genetic differences between wheat varieties but are also often caused by varying climatological conditions shortly before harvest. Untimely rainfalls after physiological maturity evoke preharvest sprouting or germination in the ear of the parent plant in the field. ${ }^{1}$ During the early stages of germination, gibberellic acid activates the production of hydrolytic enzymes in the scutellum and aleurone layer. The secreted hydrolytic enzymes are then transported to the starchy endosperm where they catalyze the hydrolysis of cell wall materials, starch, and proteins to the advantage of the growing seedling. ${ }^{1-4}$

Because of excessive hydrolytic enzyme activities in flour from PHS wheat and concomitant degradation of major and minor constituents in the seed or during production processes, the quality of several cereal-based food products can be seriously affected. For example, flour from PHS wheat produces dough that is sticky, insufficiently elastic, and difficult to handle. It results in bread with a sticky texture, low loaf volume, poor sliceability, and a darker crumb and crust color. ${ }^{5-7}$ As bakery products made of PHS wheat are generally unacceptable to producers and consumers, sprouted grains are often downgraded to feed grain or are to some extent used for other applications like gluten-starch separation and brewing. However, variation in hydrolytic enzyme activities can presumably also affect these other cereal-based food and feed applications. $^{8-10}$ Consequently, insight into the evolution of the hydrolytic enzyme activities during preharvest sprouting is of great interest when investigating methods targeted at enhancing the quality of PHS wheat.

Nevertheless, to date, there are surprisingly few reports in the literature on the evolution of hydrolytic enzyme activities of grains that have sprouted in the field. ${ }^{11,12}$ Instead, most research on the quality of PHS wheat has been conducted on sound wheat kernels germinated under laboratory conditions, which differ strongly from those encountered by wheat kernels that sprout in the ear in the field. In these controlled laboratory germination studies, decreased product quality is considered to be the net result of the action of several enzymes that degrade storage compounds prior to and during processing. The main damage to product quality is attributed to elevated $\alpha$-amylase activity levels, which result in breakdown of starch in the wheat kernel during early germination ${ }^{13,14}$ and cause further hydrolysis of damaged and gelatinized starch during fermentation and baking when using the resulting wheat flour for bread making. ${ }^{6,7}$ As a consequence, the Hagberg Falling Number (FN) method, an indirect measurement of $\alpha$-amylase activity, ${ }^{15}$ is widely used to grade wheat into quality classes. Also, elevated peptidase activity is considered as a quality-affecting factor. It is

Received: April 15, 2016

Revised: June 23, 2016

Accepted: June 24, 2016

Published: June 24, 2016 
known that, upon germination, proteolytic hydrolysis occurs in the kernel. ${ }^{4,16,17}$ This, and similar activity during processing, would lead to a reduction in gluten strength, resulting in bread doughs with an impaired gas-retaining capacity. ${ }^{4,5}$ Less information is available on the levels of other enzyme activities, such as endoxylanases, in germinating wheat. ${ }^{18,19}$ These enzymes are involved in hydrolysis of aleurone and endosperm cell walls. Accumulation of these enzymes can also be expected to affect the technological functionality of flour. ${ }^{8}$

It is difficult, however, to extrapolate these conclusions from research performed on sprouting of wheat kernels under laboratory or industrial conditions to preharvest sprouting in the field for a number of reasons. First, the conditions of temperature and humidity under which germination of wheat kernels in the field occurs are far from the ideal conditions generally applied during germination in the laboratory or in malt factories, and they result in an inefficient and nonuniform onset of germination in the field. In the second instance, this leads to a nonuniform distribution and extent of germination of the kernels in the ear (unpublished results) in contrast to a simultaneous germination of almost all kernels when germinating under laboratory conditions. It seems safe to assume that the resulting physiological changes also differ in these two kinds of germination. Indeed, Meredith and Jenkins, ${ }^{20}$ for example, observed a second important starchdegrading enzyme component in field-sprouted wheat that is lacking in laboratory-sprouted grain. It was concluded that germination of wheat in the laboratory is not representative for preharvest sprouting of wheat. ${ }^{20}$ Furthermore, characterization of field-sprouted materials is often done by counting the percentage of visually sprouted wheat kernels. However, the enzyme activity in a kernel can vary enormously depending on the severity of sprouting within the kernel; therefore, the percentage of sprouted kernels would not necessarily give a correct indication of actual levels of enzyme activity, ${ }^{6}$ making comparisons between different studies difficult. An additional issue is that in previous studies the surface of sound wheat kernels is generally disinfected prior to laboratory germination to prevent microbiological contamination. ${ }^{2,4,16,18,21}$ However, enzymes associated with wheat kernels not only originate from the wheat plant itself but also from microorganisms populating the outer layers of the wheat kernel. ${ }^{22}$ Hence, the enzyme activities measured for grain kernels sprouted in the field are also partly from microbial origin, of which the contribution is underestimated in studies using laboratory-sprouted grains.

All of the above implies that detailed knowledge on the physiological and functional changes, and more specifically on changes in hydrolytic enzyme activities, occurring during field sprouting of wheat is lacking. To have a better understanding of the factors responsible for the quality deterioration of end products made from PHS wheat flour, it is required to obtain representative PHS wheat samples in the field. In this study, the evolution of $\alpha$-amylase, $\beta$-amylase, peptidase, and endoxylanase activity in flours of three different wheat varieties (Sahara, Forum, and Tobak) as a function of harvest time and consequent sprouting in the field was investigated. A distinction between endogenous and wheat-associated microbial enzymes activities was made.

\section{MATERIALS AND METHODS}

Sample Collection and Preparation of Whole Meal and Flour. Three Belgium winter wheat (Triticum aestivum) cultivars, Forum, Tobak, and Sahara, with a difference in sprouting tolerance, were cultivated in a standard manner in 2013-2014 at the experimental site of the Universite de Liege (Agro-bio Tech, Gembloux, Belgium). Amount of rainfall and minimum and maximum temperatures were measured daily. Every 2 or 3 days, throughout the period from prematurity to extremely late maturity (July 23 until September 4), 2-3 kg of wheat grain was harvested by hand with secateurs. In this way a broad range of samples was collected that, within one wheat variety, differed in harvest time. After harvesting, samples were dried overnight at $40{ }^{\circ} \mathrm{C}$. One part of each batch of wheat was ground into whole meal with the Laboratory Mill 3100 (Perten Instruments, Hägersten, Sweden). Another part of the kernels was conditioned to $16.0 \%$ moisture and subsequently milled into flour with a Bühler MLU-202 laboratory mill (Bühler AG, Uzwil, Switzerland). The obtained flour samples consisted of three break roll flour fractions and three reduction roll flour fractions. Total flour yield ranged from 71 to $75 \%$. All wheat samples were milled within 2 months after harvest, and the resulting flours were stored at $7{ }^{\circ} \mathrm{C}$ before analysis. Kernel and flour moisture content and flour ash content were determined according to AACC International methods 44-15.02 and 08-01.01, respectively. ${ }^{23}$ Ash content ranged from 0.49 to $0.72 \%$ (on dry matter $(\mathrm{dm})$ ). The remaining part of the batch was used for the washing experiment (cf. infra). The percentage of sprouting was assessed visually by counting the amount of kernels in which the radicle and/or the coleoptile had penetrated the pericarp of 200 randomly chosen kernels. Results are expressed as percentage by count.

Chemicals and Reagents. Chemicals and reagents were purchased from Sigma-Aldrich (Bornem, Belgium) and were of at least analytical grade. Azurine-cross-linked arabinoxylan and amylose tablets were purchased from Megazyme (Bray, Ireland).

Hagberg Test. The FN of the different samples was measured in triplicate according to AACC International method $56-81.03^{23}$ and with a sample size of $7 \mathrm{~g}$ (14\% moisture basis) in $25 \mathrm{~mL}$ of $\mathrm{H}_{2} \mathrm{O}$. The FN of the flour and whole meal samples is defined as the total time in seconds required to stir (60 s) and allow a FN1500 viscosimeter stirrer to fall a specified distance through the heated flour-water suspension. ${ }^{15}$

Washing of Wheat Kernels. Washing of sound and PHS wheat kernels $(100 \mathrm{~g})$ of the different varieties was performed in triplicate as described by Dornez et al. ${ }^{22}$ with the use of universal buffer $\mathrm{pH} 8.0$ as washing liquid and a washing time of $17 \mathrm{~h}$. Universal buffer was prepared by dissolving citric acid $(6.0 \mathrm{~g})$, monopotassium phosphate $(3.9 \mathrm{~g})$, boric acid $(1.8 \mathrm{~g})$, diethyl barbituric acid $(5.3 \mathrm{~g})$, and sodium azide $(0.2 \mathrm{~g})$ in deionized water $(1.0 \mathrm{~L})$ and adjusting the $\mathrm{pH}$ with a $\mathrm{NaOH}$ solution $(2.0 \mathrm{M})$. Washed wheat kernels were ground with a Cyclotec 1093 sample mill (FOSS, Hogänäs, Sweden).

Analysis of Enzyme Activities. $\alpha$-Amylase and endoxylanase activity levels in flour, whole meal made from washed kernels, and the washing liquid were determined with the Amylazyme and Xylazyme methods, respectively (Megazyme). Enzymes were extracted by suspending $1.0 \mathrm{~g}$ of flour or meal in $10.0 \mathrm{~mL}$ of maleate buffer ( 100 $\mathrm{mM}, \mathrm{pH} 6.0$ ) containing $5 \mathrm{mM} \mathrm{CaCl}$ for the Amylazyme method and in $10.0 \mathrm{~mL}$ of sodium acetate buffer $(25 \mathrm{mM}, \mathrm{pH} 5.0)$ for the Xylazyme method. Suspensions were shaken $\left(30 \mathrm{~min}, 7{ }^{\circ} \mathrm{C}\right)$ and centrifuged $\left(4000 \mathrm{~g}, 10 \mathrm{~min}, 7^{\circ} \mathrm{C}\right)$. An azurine-cross-linked amylose or arabinoxylan tablet was added to $1.0 \mathrm{~mL}$ of pre-equilibrated flour or meal extract or washing liquid at $40{ }^{\circ} \mathrm{C}$. After appropriate incubation times, the reaction was stopped by adding $10.0 \mathrm{~mL}$ of Tris(hydroxymethyl)aminomethane solution $(2.0$ or $1.0 \mathrm{w} / \mathrm{v} \%$ for the Amylazyme or Xylazyme method, respectively). After filtration of the suspensions, the extinction values at $590 \mathrm{~nm}$ (Ultraspec 2100 pro spectrophotometer, Biochrom Ltd., Cambridge, U.K.) were measured against a control, prepared by incubating the extracts without the tablet. Correction was made for nonenzymatic color release by the substrate tablets. Activities were expressed in $\alpha$-amylase and endoxylanase units (AU and EU, respectively) per gram $\mathrm{dm}$. One unit is defined as the amount of enzyme activity needed to yield a corrected extinction value of 1.0 per hour of incubation under the conditions of the assay. 
$\beta$-Amylase activity levels in flour, whole meal made from washed kernels, and the washing liquid were determined with the Betamyl-3 method (Megazyme) according to Struyf et al. ${ }^{24}$ For the measurement of free and soluble $\beta$-amylase activity, flour $(1.0 \mathrm{~g})$ was suspended in $10.0 \mathrm{~mL}$ of TRIS-HCl buffer (0.05 M, pH 8.0) containing $1.0 \mathrm{mM}$ disodium ethylenediaminetetraacetic acid and sodium azide $(0.02 \% \mathrm{w} /$ v). To assess the total $\beta$-amylase activity, $100 \mathrm{mM}$ cysteine was added to the extraction buffer. Activities were expressed in $\beta$-amylase units (BU) per gram $\mathrm{dm}$. One $\mathrm{BU}$ is defined as the amount of enzyme activity required to release $1 \mu \mathrm{mol}$ of $p$-nitrophenol from $p$ nitrophenyl- $\beta$-D-maltotrioside, in the presence of excess $\beta$-glucosidase, per $\mathrm{g} \mathrm{dm}$ per min at $40{ }^{\circ} \mathrm{C}$ and $\mathrm{pH}$ 6.2. The amount of bound $\beta$ amylases was calculated as the difference of total and free $\beta$-amylase activity.

Exo- and endopeptidase activity levels in flour, whole meal made from washed kernels, and the washing liquid were determined using hemoglobin as substrate according to Brijs et al. ${ }^{25}$ To measure peptidase activity at different $\mathrm{pH}$ conditions, $0.2 \mathrm{M}$ sodium acetate buffer adjusted to $\mathrm{pH}$ 3.0, 4.0, and 5.0; Mcllvaine buffer ( $\mathrm{pH}$ 6.0, prepared by mixing appropriate amounts of $0.1 \mathrm{M}$ citric acid and $0.2 \mathrm{M}$ disodium phosphate); and $0.2 \mathrm{M}$ sodium phosphate buffer adjusted to $\mathrm{pH} 7.0$ and 8.0 were used. Total peptidase activity levels are expressed in peptidase units $(\mathrm{PU})$ per gram $\mathrm{dm}$, with one PU corresponding to the amount of enzyme activity needed to yield a corrected extinction value of 1.0 per hour of incubation under the assay conditions.

Enzyme extractions and subsequent enzyme activity measurements were performed in triplicate. The enzyme activities in the washing liquid were expressed as enzyme units per gram of originally treated wheat rather than per milliliter of liquid.

Analysis of Inhibition Sensitivity of Endoxylanases. To measure the inhibition sensitivity of kernel-associated and endogenous endoxylanases, endoxylanase inhibitors were extracted from mature Forum wheat flour $(1.0 \mathrm{~g}$ in $10.0 \mathrm{~mL}$ of $25 \mathrm{mM}$ sodium acetate buffer $\mathrm{pH}$ 5.0) containing high amounts of endoxylanase inhibitors but only low levels of endoxylanase. Whole meal enzyme extracts or washing liquids $(0.5 \mathrm{~mL})$ were then preincubated with the extracted inhibitors $(0.5 \mathrm{~mL})$ for $30 \mathrm{~min}$ at $40{ }^{\circ} \mathrm{C}$, allowing enzyme-inhibitor complexes to be formed. The endoxylanase activity in the preincubated samples was measured with the Xylazyme method as described above and corrected for the endoxylanase activity present in the aqueous extract of the Forum flour sample.

Statistical Analysis. For reproducibility and accuracy measurements, all (bio)chemical experiments were performed in triplicate. Significant differences were determined by one-way analysis of variance using JMP Pro software 11 (SAS Institute, Cary, NC, U.S.A.), with comparison of mean values using the Tukey test $(\alpha=$ $0.05)$.

\section{RESULTS AND DISCUSSION}

Case Description. In the present study, a broad set of wheat samples, which differ in harvest time within one wheat variety, was collected to obtain a better understanding of the evolution of hydrolytic enzyme activity as a function of harvest time. The daily amount of rainfall, daily temperatures, and FN of whole meal were measured to follow up the sprouting process during the summer of 2014 (Figure 1). Initially (July 23), FN of the whole meal samples increased slightly, indicating that harvest maturity was not yet reached at the beginning of wheat sample collection. In general, at maximal FN values harvest maturity is assumed to be reached, which is associated with a reduction in moisture content of the kernel ${ }^{26}$ and a drop of the $\alpha$-amylase activity in the outer layers of the kernel..$^{27,28}$ For the selected data set, harvest maturity was obtained between the last days of July and the first week of August with a maximal whole meal FN of 245,339 , and 330 s for Sahara, Forum, and Tobak, respectively (Figure 1).
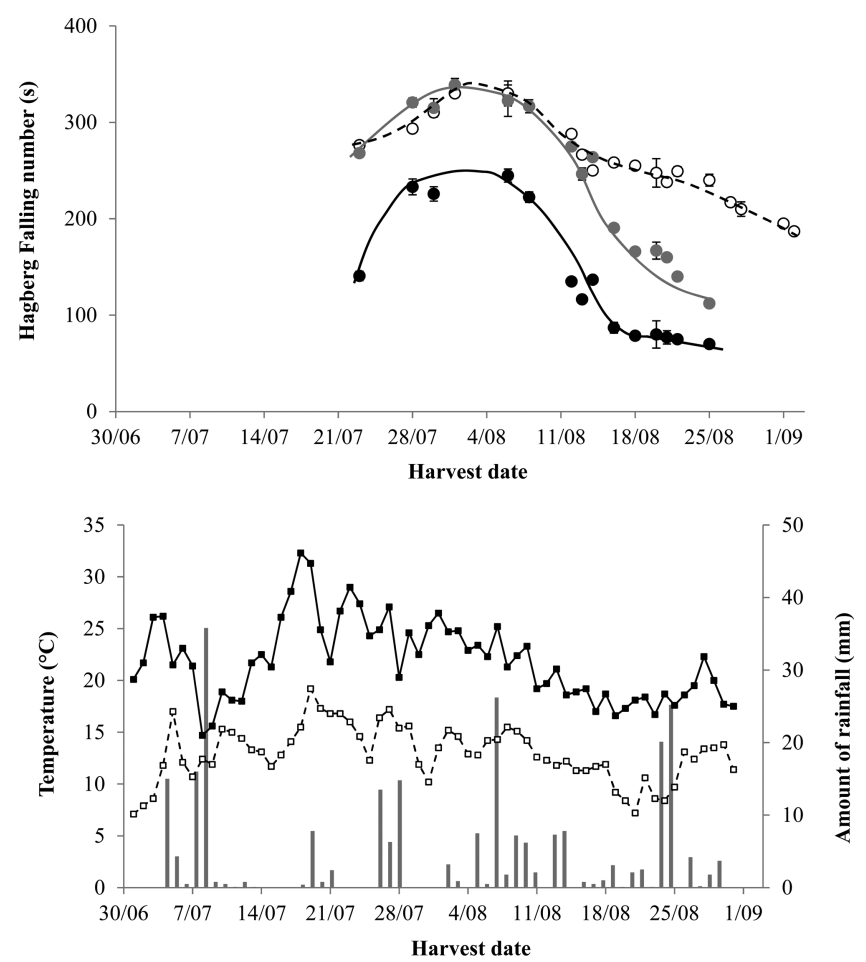

Figure 1. (Top) Hagberg Falling Number (s) of whole meal of Sahara (black spheres), Forum (gray spheres), and Tobak (white spheres) as a function of harvest time. Values are averages with standard deviations on triplicate measurements. (Bottom) Daily amount of rainfall (mm) during harvest time indicated as gray bars and minimum and maximal daily temperature $\left({ }^{\circ} \mathrm{C}\right)$ indicated as white and black squares, respectively.

According to Mares, ${ }^{29}$ repeated wetting and drying cycles can lead to swelling and shrinking of the grains and trigger the mature grain to break its dormancy and make the kernel more susceptible to preharvest sprouting in the presence of suitable germination conditions. During the summer of 2014, periods of heavy rainfall around the 5th, 20th, and 27th of July (Figure 1) caused wheat grains to lose their dormancy and, hence, sprouting in the ear occurred, which was visible in some kernels of the sprouted samples as the coleoptile and/or radicle had penetrated the pericarp (Figure S1 in Supporting Information). This was concomitant with a decrease in FN when harvest time of the matured wheat grain was delayed. Rainy weather conditions and the occurrence of visible sprouting indicated that preharvest sprouting is the dominant cause of the decrease in FN rather than other phenomena like late maturity $\alpha$ amylase activity, which also gives rise to high $\alpha$-amylase activity levels in wheat. ${ }^{30}$ Differences in the magnitude of the decline in FN suggest that the three varieties differ in susceptibility toward preharvest sprouting. Sahara wheat samples were characterized by an overall low FN throughout the entire harvest period, and after harvest maturity a fast drop in FN was observed (value of $70 \mathrm{~s}$ at August 25), which shows that Sahara was sensitive toward preharvest sprouting. At that moment $10.5 \%$ of the kernels were visibly sprouted by penetration of the coleoptile. Compared to Sahara, the FN of Forum samples evolved similarly as a function of harvest time, although the initial FN was higher and dropped only to $112 \mathrm{~s}$. The smaller extent of sprouting of Forum was also noticed in the lower percentage of visible sprouted kernels (6\%). The Tobak variety, on the contrary, appeared to be the most resistant variety toward 
preharvest sprouting as only $1 \%$ of the kernels were visibly sprouted and the drop in FN occurred slower when harvest time was delayed under the same climatological conditions until a value of $240 \mathrm{~s}$ at August 25. Differences in preharvest sprouting susceptibility between wheat varieties can be explained by their genetic background as this determines the level of dormancy and the sensitivity of a wheat kernel to break out of its dormancy in the presence of certain environmental conditions. ${ }^{1}$

$\alpha$-Amylase Activity. $\alpha$-Amylase Activity of Flour as a Function of Harvest Time. In this study, the $\alpha$-amylase activity in flour derived from the harvested kernels was assessed in two ways. On the one hand the Hagberg FN test was used, as this indirectly measures $\alpha$-amylase activity, ${ }^{15}$ and on the other hand the activity was measured directly using a dyed and cross-linked amylose substrate (Amylazyme method). Between these two parameters, a strong logarithmical relationship was found $\left(R^{2}=\right.$ 0.99, 0.98, and 0.96 for Sahara, Forum, and Tobak, respectively) (Figure 2), as also reported earlier. ${ }^{31}$ In Figure

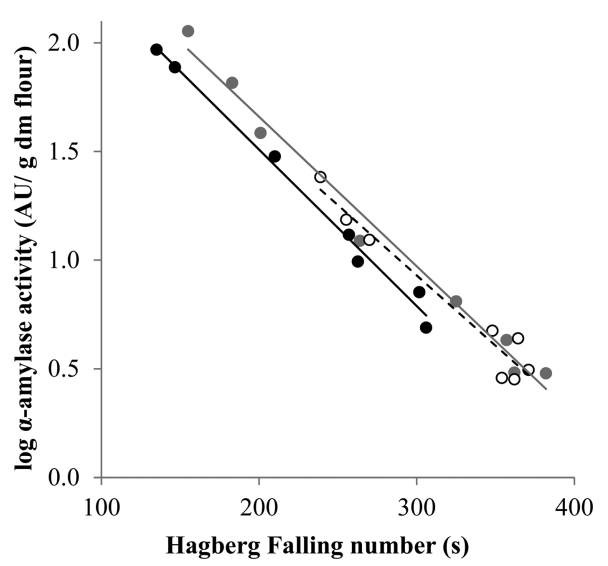

Figure 2. Logarithmic relationship between Hagberg Falling Number (s) and $\alpha$-amylase activity of flour samples of Sahara (black spheres), Forum (gray spheres), and Tobak (white spheres) harvested between July 23 and September 2. $\alpha$-Amylase activity was defined in $\alpha$-amylase activity units (AU) per g of flour dry matter (dm).

3 the $\alpha$-amylase activity and FN of flour are represented as a function of harvest time. For FN, in general, the same trends were observed as those detected for the corresponding whole meal samples (Figures 1 and 3), but the FN of flour of the different wheat varieties was characterized by a higher value. This higher value was caused by the removal of bran material containing relatively higher $\alpha$-amylase levels during milling. ${ }^{27}$ However, differences between whole meal FN and flour FN were not constant for different varieties and at different harvest time points, indicating that the FN of whole meal is not a very accurate predictor for $\mathrm{FN}$ of flour.

Before harvest maturity was reached, $\alpha$-amylase activity slightly decreased from 10 to $5 \mathrm{AU} / \mathrm{g}$ in flour of the Sahara variety, which corresponds to an increase in FN from 263 to $307 \mathrm{~s}$ (Figure 3). The increase in FN of flour from Forum and Tobak was less pronounced, and correspondingly no significant decrease in $\alpha$-amylase activity was detected when harvest maturity was achieved. The $\alpha$-amylase activity in these samples was initially already very low (3 AU/g for both Forum and Tobak), suggesting that the drop in enzyme activity, typically when maturation progresses, ${ }^{27,28}$ had occurred before the start of harvest in this study. Delaying harvest after harvest maturity resulted in a strong increase in $\alpha$-amylase activity, which was more pronounced as harvest time was further postponed until August 20 and reached values of 93 and $65 \mathrm{AU} / \mathrm{g}$ in flours of Sahara and Forum, respectively. This rise in $\alpha$-amylase activity was accompanied by a strong decline in FN to values of 147 and $183 \mathrm{~s}$ in the flour samples of Sahara and Forum, respectively. While the initial slow increase in $\alpha$-amylase activity is rather due to the disappearance of $\alpha$-amylase inhibitors as germination sets on, ${ }^{32}$ the large changes in $\alpha$ amylase activity of flour are characteristic features of preharvest sprouting and are attributed to de novo synthesis of two groups of isoenzymes, low and high pI $\alpha$-amylases. ${ }^{2,3}$ Due to periods of heavy rainfall, the increase in $\alpha$-amylase activity over a one month period-namely, a 19-, 22-, and 4-fold rise for Sahara, Forum, and Tobak, respectively-was larger than that in the study of Noda et al., ${ }^{11}$ in which a similar time span was examined, indicating the importance of weather conditions for preharvest sprouting to occur. Several other authors have been studying the development of $\alpha$-amylase activity during the germination process in the laboratory and noticed a several hundred fold increase after 4 or 5 days of germination. ${ }^{11,18,21}$ However, these experiments were performed on mature wheat kernels germinated under optimal conditions in the laboratory, which most likely resulted in a homogeneous and immediate onset of germination and, hence, more pronounced changes in enzyme activity, and results can therefore not be transferred as such to field sprouting.

The fast increase in $\alpha$-amylase activity in the flour samples of Sahara again confirms the low resistance of this variety toward preharvest sprouting. Compared to Sahara and Forum, the rise in $\alpha$-amylase activity and concomitant decline in FN of Tobak flour was much more gradual and less pronounced, as already indicated by the FN results of the whole meal samples (Figure $1)$. The FN values of the sample harvested at August 20 reached a value of $268 \mathrm{~s}$, whereas the $\alpha$-amylase activity detected was $13 \mathrm{AU} / \mathrm{g}$, suggesting a higher resistance of Tobak toward preharvest sprouting.

Distribution of $\alpha$-Amylase Activity over the Kernel. Enzymes associated with wheat kernels originate not only from the wheat plant itself but also from microorganisms populating the outer layers of the wheat kernel. ${ }^{22}$ Therefore, it was investigated to which extent endogenous enzymes contributed to the elevated $\alpha$-amylase activity levels detected in sprouted flour samples. The $\alpha$-amylase activity in the washing liquid was considered as kernel-associated $\alpha$-amylase activity, which includes microbial $\alpha$-amylase activity and possibly also $\alpha$-amylase activity originating from the pericarp. During the washing procedure slight abrasion of the kernels occurred, and hence migration of these enzymes out of the pericarp could not be prevented. No microorganisms grew during this experiment due to the presence of sodium azide, and longer washing times (up to $46 \mathrm{~h}$ ) did not lead to elevated $\alpha$-amylase activity in the washing liquid (results not shown), indicating that no enzymes were formed de novo during the washing step. The $\alpha$-amylase activity measured in whole meal made from the washed wheat kernels is regarded as endogenous $\alpha$-amylase activity. The $\alpha$-amylase activity of a mature wheat kernel predominantly consisted of endogenous $\alpha$-amylases, namely, 91\%, 90\%, and 90\% of the total $\alpha$-amylase activity for Sahara, Forum, and Tobak, respectively (Table 1). Consequently, the amount of $\alpha$-amylases that migrated from the pericarp during the treatment is rather limited. Next, these results show that whole meal endogenous $\alpha$-amylase activity 

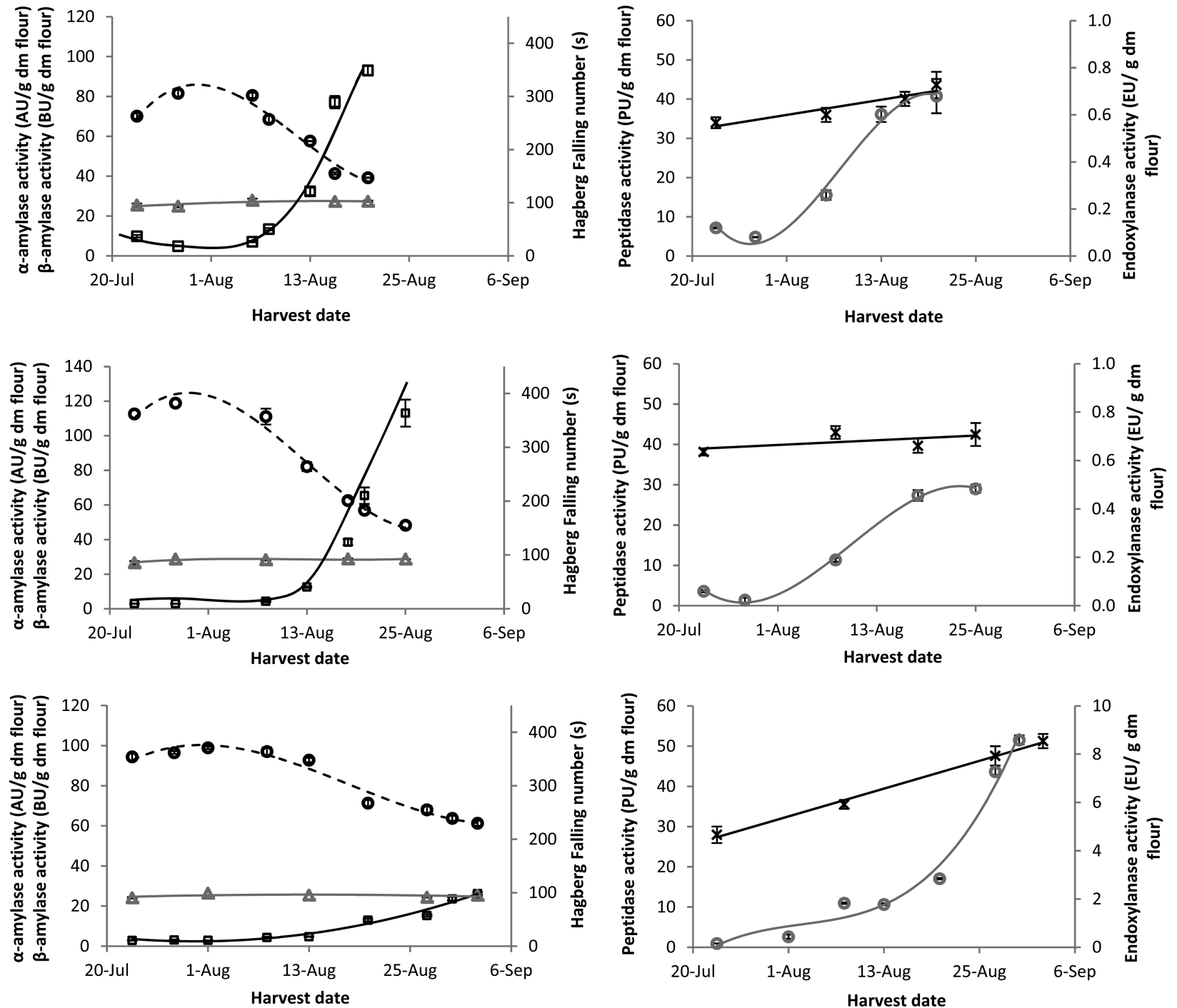

Figure 3. (Left) Overview of $\alpha$-amylase activity (squares), total $\beta$-amylase activity (triangles), and Falling Number(s) (black spheres) measured in flour of Sahara (top), Forum (middle), and Tobak (bottom) grains harvested at different dates during harvest time. (Right) Overview of peptidase activity (crosses) and endoxylanase activity (gray spheres) measured in flour of Sahara (above), Forum (middle), and Tobak (below) grain harvested at different dates during harvest time. $\alpha$-Amylase, total $\beta$-amylase, peptidase, and endoxylanase activity was defined in $\alpha$-amylase activity units (AU), $\beta$-amylase activity units (BU), peptidase activity units (PU), and endoxylanase activity units (EU) per g of flour dry matter (dm), respectively. Error bars are standards deviations on triplicate measurements.

Table 1. Endogenous and Kernel-Associated $\alpha$-Amylase Activity of Mature and Preharvest-Sprouted Kernels of Sahara, Forum, and Tobak ${ }^{a}$

\begin{tabular}{lccccc} 
& \multicolumn{2}{c}{$\begin{array}{c}\text { endogenous } \alpha \text {-amylase } \\
\text { activity }\end{array}$} & & \multicolumn{2}{c}{$\begin{array}{c}\text { kernel-associated } \alpha \text {-amylase } \\
\text { activity }\end{array}$} \\
\cline { 2 - 3 } \cline { 5 - 6 } \cline { 5 - 6 } & $(\mathrm{AU} / \mathrm{g} \mathrm{dm})$ & $(\%$ of total $)$ & & $(\mathrm{AU} / \mathrm{g} \mathrm{dm})$ & $(\%$ of total $)$ \\
Sahara 28/7 & $16 \pm 7$ & $91 \%$ & & $1.6 \pm 0.0$ & $9 \%$ \\
Sahara 20/8 & $187 \pm 8$ & $98 \%$ & & $4.4 \pm 1.1$ & $2 \%$ \\
Forum 28/7 & $5 \pm 1$ & $90 \%$ & & $0.5 \pm 0.2$ & $10 \%$ \\
Forum 25/8 & $161 \pm 6$ & $95 \%$ & & $7.8 \pm 0.9$ & $5 \%$ \\
Tobak 1/8 & $6 \pm 2$ & $90 \%$ & & $0.6 \pm 0.0$ & $10 \%$ \\
Tobak 2/9 & $39 \pm 2$ & $85 \%$ & & $6.9 \pm 0.2$ & $15 \%$
\end{tabular}

${ }^{a}$ Values are averages with standard deviations on triplicate measurements. $\alpha$-Amylase activity is expressed as $\alpha$-amylase activity units (AU) per $g$ of meal dry matter $(\mathrm{dm})$.

levels are significantly higher than flour $\alpha$-amylase activity levels (comparing Table 1 and Figure 3), indicating that these enzymes are predominantly present in the outer layers of a wheat kernel.

In addition, because of preharvest sprouting in the field, the amount of endogenous $\alpha$-amylase activity in Sahara and Forum increased 12 and 31 times to values of 187 and $161 \mathrm{AU} / \mathrm{g}$, respectively, demonstrating the de novo synthesis of these enzymes during field sprouting. The rise in endogenous $\alpha$ amylase activity in meal of Tobak was less pronounced (a 7-fold increase), which was expected based on the earlier observations. Moreover, delaying harvest resulted also in an increase in kernel-associated $\alpha$-amylase activity up to values of $4.4,7.8$, and 6.9 AU/g for Sahara, Forum, and Tobak, respectively, as heavy rainfall increases the risk not only of sprouting but also of microbial growth on the wheat kernel. However, for Sahara and Forum, this increase was small in comparison with the rise in endogenous $\alpha$-amylase activity.

$\boldsymbol{\beta}$-Amylase Activity. The total $\beta$-amylase activity levels varied around 26,27 , and $25 \mathrm{BU} / \mathrm{g}$ over the harvesting period for Sahara, Forum, and Tobak, respectively (Figure 3). No 
changes in total $\beta$-amylase activity were detected as a function of harvest time, indicating that, in contrast to $\alpha$-amylases, no de novo formation occurred during field sprouting of wheat grain until flour FN of ca. $150 \mathrm{~s}$, similar to what was previously observed for laboratory-germinated grains. ${ }^{2,21}$ De novo synthesis of $\beta$-amylases only occurs during development of the grain, ${ }^{28}$ confirming that in this study this stage was already completed before the start of sampling of wheat from the field. As almost no $\beta$-amylase activity could be detected in the washing liquid after completion of the washing procedure (results not shown), these enzymes are almost exclusively present endogenously ( $>99 \%)$, more precisely in the endosperm. The latter follows from the fact that total $\beta$-amylase activity levels measured in flour and whole meal were equal, respectively, 24.8 and $25.7 \mathrm{BU} / \mathrm{g}$ for mature flour of Sahara.

$\beta$-amylases in wheat are present in a "free and soluble" and in a "bound" form, where the latter are associated with each other or with glutenins by disulfide bonds, and require the addition of reducing agents or proteolytic enzymes for extraction and activity. ${ }^{33}$ It was observed that mature wheat kernels already contained high levels of free $\beta$-amylase activity, namely, 85,81 , and $83 \%$ of the total $\beta$-amylase activity for flour of Sahara, Forum, and Tobak, respectively. Moreover, the ratio of boundto-free $\beta$-amylases remained constant as a function of harvest time; hence, no release of bound $\beta$-amylase into its free and active form was detected in these samples upon sprouting until FN of ca. $150 \mathrm{~s}$ (Table S1 in Supporting Information). This is in contrast to most literature data on laboratory-sprouted wheat grains, where an apparent increase in $\beta$-amylase activity was detected due to progressive activation of the bound enzymes by cleavage of disulfide bonds, and this already after 2 days of germination. ${ }^{2,3,33}$ In these kernels, however, the germination process has progressed further due to the optimal conditions for germination of wheat kernels in the lab. This suggests that the release of bound $\beta$-amylases probably occurs at a later stage during germination in the field. Although $\beta$-amylase activity is responsible for the release of maltose from the nonreducing end of starch polymers, an increase in free and active $\beta$-amylase activity would probably not impact the amount of fermentable sugars for the yeast during the fermentation process, as flour contains an excess of $\beta$-amylase activity and research by Struyf et $\mathrm{al}^{24}$ indicated that supplementation of $\beta$-amylase had no effect on fermentation speed.

Endoxylanase Activity. Endoxylanase Activity of Flour as a Function of Harvest Time. Besides amylases, ungerminated wheat also contains endogenous endoxylanases. ${ }^{22,34}$ Indeed, the first harvested samples, before harvest maturity, already contained some endoxylanase activity (Figure 3), which in the flour of the Sahara and Forum varieties decreased to lower activity levels ( 0.08 and $0.02 \mathrm{EU} / \mathrm{g}$, respectively) as maturity was reached and their function becomes of less importance. In contrast, flour of Tobak was already from the beginning of sampling characterized by a higher endoxylanase activity level $(0.2 \mathrm{EU} / \mathrm{g})$ that doubled toward maturity.

Postponing harvest time after harvest maturity caused a rapid increase in endoxylanase activity up to values of $0.7,0.5$, and 2.8 EU/g for Sahara, Forum, and Tobak, respectively, harvested at August 20. During germination of cereal kernels under laboratory conditions, endoxylanase activity levels also increased, starting from an initially very low value for the first 3 days, which then increased rapidly on the fourth and fifth days of germination. ${ }^{18,19,21}$ The endoxylanase activity levels in Tobak flour were significantly higher than the ones in sprouted wheat flour of Sahara and Forum. Differences in endoxylanase activity levels between wheat varieties were expected as both genotype and climatological conditions markedly determine this variability. ${ }^{8}$ Nevertheless, it was assumed that the activity present in Tobak flour (8.6 EU/g on August 30) was too high to be only from endogenous origin, especially when taking into account the low degree of sprouting. Indeed, when inspecting the individual Tobak kernels, marks characteristic for a Fusarium infection ${ }^{35}$ were spotted as pink dots on the grain. These marks were already visible in the early harvested grain but became more abundant on the later harvested kernels. This plant pathogenic fungus produces extracellular hydrolytic cell wall degrading enzymes not only to obtain nutrients but also for penetration and infection of the host. ${ }^{35}$ Besides the typical features of this infection, the flour of Tobak was also characterized by elevated concentrations of deoxynivalenol (200 and $2000 \mathrm{ppb}$ in flour of mature and sprouted wheat, respectively), a mycotoxin associated with several Fusarium pathogens. The intensity of the Fusarium infection was only that severe in Tobak and hardly present in the other cultivars. As heavy rainfalls occurred during the sampling period, also other microorganisms could have infected wheat kernels on the field. However, to the best of our knowledge, this was not the case.

Distribution of Endoxylanase Activity over the Kernel. To investigate to which extent microbial enzymes contributed to the elevated endoxylanase activity in the different grain samples, the endoxylanase activity was measured in washing liquid and whole meal of the washed kernels. As most endoxylanase activity in the washing liquid (ca. 90\%) was inhibited by the wheat flour extract containing endoxylanase inhibitors, the kernel-associated endoxylanases were considered to be from microbial origin. The endoxylanase activity in the whole meal made from washed kernels was regarded as from endogenous origin, since this activity did not diminish in the presence of endoxylanase inhibitors (results not shown).

Table 2 shows that the majority of endoxylanase activity in a mature grain kernel comes from kernel-associated microbial

Table 2. Endogenous and Kernel-Associated Endoxylanase Activity of Mature and Preharvest-Sprouted Kernels of Sahara, Forum, and Tobak ${ }^{a}$

\begin{tabular}{lccccc} 
& \multicolumn{2}{c}{$\begin{array}{c}\text { endogenous endoxylanase } \\
\text { activity }\end{array}$} & & \multicolumn{2}{c}{$\begin{array}{c}\text { kernel-associated } \\
\text { endoxylanase activity }\end{array}$} \\
\cline { 2 - 3 } \cline { 5 - 6 } \cline { 5 - 6 } & $(\mathrm{EU} / \mathrm{g} \mathrm{dm})$ & $(\%$ of total $)$ & & $(\mathrm{EU} / \mathrm{g} \mathrm{dm})$ & $(\%$ of total) \\
Sahara 28/7 & $0.3 \pm 0.01$ & $10 \%$ & & $2.7 \pm 0.2$ & $90 \%$ \\
Sahara 20/8 & $1.6 \pm 0.11$ & $7 \%$ & & $23.2 \pm 1.8$ & $93 \%$ \\
Forum 28/7 & $0.1 \pm 0.01$ & $4 \%$ & & $2.4 \pm 0.1$ & $96 \%$ \\
Forum 25/8 & $0.8 \pm 0.03$ & $3 \%$ & & $28.2 \pm 0.2$ & $97 \%$ \\
Tobak 1/8 & $1.9 \pm 0.13$ & $6 \%$ & & $29.5 \pm 3.2$ & $94 \%$ \\
Tobak 2/9 & $8.1 \pm 0.17$ & $9 \%$ & & $84.8 \pm 1.9$ & $91 \%$
\end{tabular}

${ }^{a}$ Values are averages with standard deviations on triplicate measurements. Endoxylanase activity is expressed as endoxylanase activity units (EU) per g of meal dry matter (dm)

endoxylanases $(90,96$, and 94\% for Sahara, Forum, and Tobak, respectively), which is opposite to the distribution observed for $\alpha$-amylase activities (cf. Table 1). When delaying harvest, endogenous endoxylanase levels in the samples increased and this increase was larger for Sahara and Forum than for Tobak, as sprouting had occurred less in the last variety. Hence, during sprouting in the field endoxylanases are also formed de novo in 
addition to $\alpha$-amylases. Dornez et al. ${ }^{12}$ also noticed that endogenous endoxylanase activity levels were affected by harvest date and weather conditions prior to harvest and noted a strong correlation between FN, $\alpha$-amylase activity levels, and endogenous endoxylanase levels. In addition, the occurrence of preharvest sprouting was also accompanied by a major increase in microbial endoxylanase activity (approximately a 9-, 12-, and 3-fold increase for Sahara, Forum, and Tobak, respectively).

At last, surprisingly, not only were the microbial endoxylanase activities in Tobak wheat samples high, but also the endogenous endoxylanase activity levels, compared to Sahara and Forum. This could not be explained by insufficient removal of the microbial enzymes from the wheat kernel (results not shown) nor was it due to penetration of fungal enzymes in the kernel as the activity was not reduced after incubation with endoxylanase inhibitors ( 8.0 and $8.1 \mathrm{EU} / \mathrm{g}$ in the absence and presence of inhibitors, respectively). Dornez et al. ${ }^{12}$ also detected increased endogenous endoxylanase activities in wheat samples with elevated microbial contamination when fungicide treatment was omitted compared to when fungicides were used. As the sprouting process had not progressed as far in Tobak as in the other varieties, one may speculate that a certain plant defense mechanism exists that causes an increase in endogenous endoxylanase activity as a response to Fusarium infection.

Peptidase Activity. On the basis of their catalytic mechanism, most peptidases can be divided into four classes: aspartic, metallo, cysteine, and serine peptidases. Each group is characterized by class-specific inhibitors and an optimal $\mathrm{pH}$ range. ${ }^{16}$ To study which kind of peptidases are mostly present or formed during field sprouting of wheat, the peptidase activity in PHS flour of Forum ( $\mathrm{FN}=155 \mathrm{~s}$ ) was measured at different $\mathrm{pH}$ conditions. Figure 4 shows that the highest peptidase

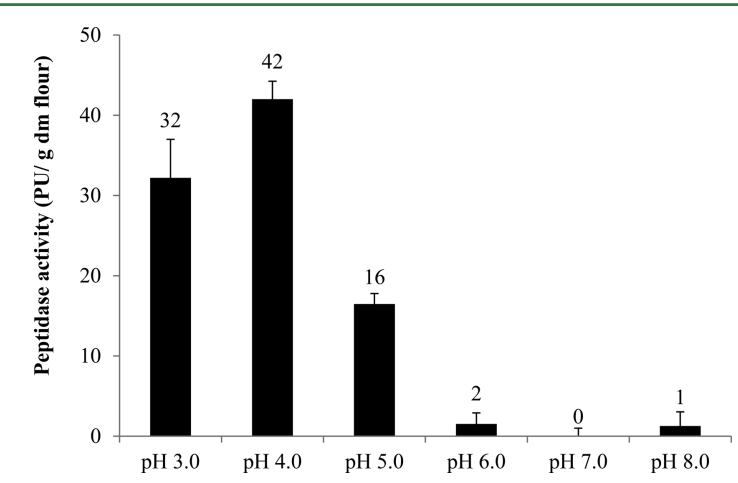

Figure 4. Peptidase activity in preharvest sprouted flour of Forum (harvested at August 25) measured under different $\mathrm{pH}$ conditions (from 3.0 to 8.0 ). Peptidase activity was defined in peptidase activity units (PU) per $\mathrm{g}$ of flour dry matter $(\mathrm{dm})$. Values are averages with standard deviations on triplicate measurements.

activity was present under acidic conditions, 32 and $42 \mathrm{PU} / \mathrm{g}$ for $\mathrm{pH} 3.0$ and 4.0, respectively, which suggests the presence of aspartic and acid cysteine peptidases. The presence of these kind of peptidases in germinated grain was also observed by Dominguez and Cejudo ${ }^{16}$ in wheat and by several authors in rye and barley. ${ }^{25,36}$ In the present study, hardly any peptidase activity was detected at neutral to alkali $\mathrm{pH}$ values, which is in contrast to earlier studies describing the presence of neutral cysteine peptidases and alkali serine peptidases in germinated grain. ${ }^{17,25,36}$ This again indicates that sprouting in the field appears to stimulate the production of hydrolytic enzymes in a different manner than under laboratory conditions. The present results suggest, hence, that the impact of sprouting on the gluten properties of flour will be smaller in nonacidic production processes, such as the early stage of bread making, compared to what was considered earlier. Instead, due to the more acidic conditions ruling during gluten-starch separation, the elevated peptidase activity may have a more detrimental effect on the quality of the obtained gluten. Nonetheless, in this context, Kelfkens and Hamer ${ }^{9}$ noted earlier that moderately sprout damaged wheat $(\mathrm{FN} \approx 150 \mathrm{~s})$ can still be used in starch-gluten fractionation processes with no effect on gluten yield and quality; the gluten agglomeration was even improved due to the increased activity of endoxylanases.

When differentiating endogenous from kernel-associated peptidases, no peptidase activity could be detected in the washing liquid. Comparing peptidase activity of whole meal from washed kernels $(66 \mathrm{PU} / \mathrm{g}$ ) with activity measured in flour (42 PU/g), the findings indicated that in PHS wheat these enzymes are for $56 \%$ present in bran layers and germ removed by milling and for $44 \%$ present in the produced flour.

On the basis of the above results, peptidase activity was measured for all samples at $\mathrm{pH} 4.0$ to study changes in peptidase activity as a function of harvest time (Figure 3). Flour of wheat kernels that almost reached harvest maturity already contained 34, 38, and 28 PU/g for Sahara, Forum, and Tobak, respectively. Although all varieties showed a slight increase in acidic peptidase activity as a function of harvest time to values of 44, 42, and $51 \mathrm{PU} / \mathrm{g}$ in flour of Sahara, Forum, and Tobak, respectively, these increases were much lower than expected, based on the (large) drop in FN values during this period. It is even possible that such an increase in peptidase activity is due to removal of proteolytic inhibitors rather than due to synthesis of new proteins. ${ }^{17}$ These results show that de novo formation of $\alpha$-amylases and peptidases is regulated very differently and, hence, that FN values cannot predict peptidase activity levels. Compared to the other two varieties, peptidase activity increased much more for the Tobak variety, which was remarkable, knowing that $\alpha$-amylase activity levels only slightly increased in comparison to Sahara and Forum. Probably the Fusarium infection contributed to the elevated peptidase activities, as fungi also require peptidases for complete digestion of plant cell walls and as a nitrogen source. ${ }^{35}$ Previous research on laboratory-sprouted grain showed that the acidic peptidase activity largely increased upon germination due to de novo formation of enzymes. Most studies detected an initial small increase in peptidase activity after $24 \mathrm{~h}$, followed by a 5- to 10 fold rise after $4-5$ days of germination. ${ }^{4,5,16,25,36}$ As explained above, it seems logical that the increase in peptidase activity was less pronounced in this study as the wheat grain experiences less ideal conditions for germination in the field than in the laboratory.

Relevance of the Present Findings. The present study clearly demonstrates that unfavorable weather conditions around harvest time markedly influence hydrolytic enzyme activity levels in a manner distinct from what was previously observed for sound wheat kernels germinated under laboratory conditions as a proxy for field sprouting. Preharvest sprouting differs quantitatively from laboratory sprouting due to less ideal conditions and inefficient onset of germination in the field, inducing a slow and less pronounced increase in hydrolytic enzyme activities. In addition, qualitative differences were observed that are the results of the nonuniform distribution and 
extent of germination of the kernels in the ear. Nevertheless, due to a lack of data on field-sprouted wheat, the results from laboratory germination studies are frequently used to predict the impact of field sprouting on end product quality.

Moreover, the present investigation also reveals that the regulation of the different hydrolytic enzymes studied here during field sprouting differed temporally. A first observation was that the acidic peptidase activity increased only slightly and that active $\beta$-amylase did not, although $\mathrm{FN}$ values were considerably lower. In addition, the rise in endoxylanase activity started markedly earlier than the increase in $\alpha$-amylase activity and the concomitant drop in FN. This is in contrast to previous reports on germinative enzymes, ${ }^{18,21}$ in which it was logically assumed that the production of cell wall degrading enzymes was a critical step as these cell walls construct a physical barrier for other endogenous hydrolyzing enzymes to access and degrade intracellular starch and protein. ${ }^{18,27}$ However, as the endoxylanase activity is predominantly coming from microbial enzymes associated with the kernel, unfavorable weather conditions like the heavy rainfalls of July 27-29 (Figure 1) will cause a fast microbial growth and, hence, an increase in microbial endoxylanase levels associated with the kernel. The increase in $\alpha$-amylase activity is more delayed because of the time needed for the kernel to break dormancy and start to synthesize $\alpha$-amylases after sufficient water imbibition. This indicates that $\alpha$-amylase activity does not correlate well with $\beta$ amylase, peptidase, and endoxylanase activities measured in flour and that FN cannot be used as sole criterion to predict the degree of sprout damage in wheat.

In addition, the present findings highlight the importance of the distinction between endogenous enzymes and kernelassociated enzymes. While the $\alpha$-amylase activity of wheat kernels was predominantly of endogenous origin and situated in the outer layers, the endoxylanase activity was dominated by microbial enzymes associated with the kernel surface. In previous studies unravelling the causes of deteriorated product quality due to PHS wheat, the contribution of enzymes from microorganisms associated with the grain kernel was underestimated. However, as part of these enzymes will end up in flour after milling and, hence, can affect end product quality, the presence of microbial endoxylanases in cereal processing should not be neglected. In this context, Gys et al. ${ }^{37}$ showed that kernel-associated microbial endoxylanases were involved in syruping of dough during storage.

It should also be mentioned that the influence of a particular enzyme during cereal processing will depend on whether the conditions are suitable for its action or not. It is noteworthy that the changes detected in the present study were observed under optimal operation conditions for each specific enzyme. For example, temperature, $\mathrm{pH}$ value, moisture content, substrate access, etc. might be different and less ideal in a real food system. Hence, the relative importance of different enzymes will vary from application to application.

In conclusion, germination in the field appears to stimulate hydrolytic enzyme production in a quantitatively and qualitatively different manner than sprouting under laboratory conditions. Consequently, more care should be taken when using results of laboratory germination experiments to predict quality-changing aspects from PHS flour. Nevertheless, further research with a focus on technological aspects is required to completely understand the effect of these enzymes in PHS wheat on end product quality. This knowledge will contribute to finding remedies to reduce the detrimental impact of the increased enzyme activity during processing. In this context, pearling of the wheat grain before milling can be a promising pretreatment to enhance flour quality from PHS wheat, as pearling can greatly reduce the surface-associated enzyme activities and remove a substantial amount of the enzyme-rich bran and germ.

\section{ASSOCIATED CONTENT}

\section{Supporting Information}

The Supporting Information is available free of charge on the ACS Publications website at DOI: 10.1021/acs.jafc.6b01711.

Photographs of a selection of mature and field-sprouted wheat kernels of Sahara, Forum, and Tobak; measured free and bound $\beta$-amylase activities in flours of Sahara, Forum, and Tobak wheat harvested at different dates during harvest time (PDF)

\section{AUTHOR INFORMATION}

\section{Corresponding Author}

*Phone: +32 $16 \quad 3219$ 17. E-mail: christophe.courtin@ kuleuven.be.

\section{Funding}

H.O. acknowledges Flanders' FOOD (Brussels, Belgium) for financial support within the framework of the REDUxAMYL project.

\section{Notes}

The authors declare no competing financial interest.

\section{ACKNOWLEDGMENTS}

The authors are grateful to Luc Van den Ende for the technical support.

\section{ABBREVIATIONS USED}

AU, $\alpha$-amylase activity units; BU, $\beta$-amylase activity units; EU, endoxylanase activity units; $\mathrm{dm}$, dry matter; FN, Falling Number; PHS, preharvest sprouted; PU, peptidase activity units

\section{REFERENCES}

(1) Meredith, P.; Pomeranz, Y. Sprouted grain. In Advances in Cereal Science and Technology, 7th ed.; Pomeranz, Y., Ed.; AACC: St. Paul, MN, 1985; pp 239-320.

(2) Daussant, J.; Corvazier, P. Biosynthesis and modifications of $\alpha$ and $\beta$-amylases in germinating wheat seeds. FEBS Lett. 1970, 7 (2), 191-194.

(3) Kruger, J. Changes in the amylases of hard red spring wheat during germination. Cereal Chem. 1972, 49, 391-398.

(4) Preston, K.; Dexter, J. E.; Kruger, J. Relationship of exoproteolytic and endoproteolytic activity to storage protein hydrolysis in germinating durum and hard red spring wheat. Cereal Chem. 1978, 55 (6), 877-888.

(5) Hwang, P.; Bushuk, W. Some changes in the endosperm proteins during sprouting of wheat. Cereal Chem. 1973, 50, 147-160.

(6) Ibrahim, Y.; D'Appolonia, B. Sprouting in hard red spring wheat. Bakers Digest 1979, 53 (5), 17-19.

(7) Kozmin, N. Biochemical characteristics of dough and bread from sprouted grain. Cereal Chem. 1933, 10, 420-436.

(8) Dornez, E.; Gebruers, K.; Delcour, J. A.; Courtin, C. M. Grainassociated xylanases: occurrence, variability, and implications for cereal processing. Trends Food Sci. Technol. 2009, 20 (11), 495-510.

(9) Kelfkens, M.; Hamer, R. J. Agronomic factors related to the quality of wheat for the starch industry. Part I: Sprout damage. Starch Stärke 1991, 43 (9), 340-343. 
(10) Bamforth, C. W. Current perspectives on the role of enzymes in brewing. J. Cereal Sci. 2009, 50 (3), 353-357.

(11) Noda, T.; Ichinose, Y.; Takigawa, S.; Matsuura-Endo, C.; Abe, H.; Saito, K.; Hashimoto, N.; Yamauchi, H. The pasting properties of flour and starch in wheat grain damaged by $\alpha$-amylase. Food Sci. Technol. Res. 2003, 9 (4), 387-391.

(12) Dornez, E.; Gebruers, K.; Joye, I. J.; De Ketelaere, B.; Lenartz, J.; Massaux, C.; Bodson, B.; Delcour, J. A.; Courtin, C. M. Effects of fungicide treatment, $\mathrm{N}$-fertilisation and harvest date on arabinoxylan, endoxylanase activity and endoxylanase inhibitor levels in wheat kernels. J. Cereal Sci. 2008, 47 (2), 190-200.

(13) Dronzek, B. L.; Hwang, P.; Bushuk, W. Scanning electron microscopy of starch from sprouted wheat. Cereal Chem. 1972, 49, 232-239.

(14) MacGregor, A. W.; Matsuo, R. R. Starch degradation in endosperms of barley and wheat kernels during intial stages of germination. Cereal Chem. 1982, 3, 210-216.

(15) Perten, H. Application of the Falling number method for evaluating $\alpha$-amylase activity. Cereal Chem. 1964, 41 (3), 127-139.

(16) Dominguez, F.; Cejudo, F. J. Pattern of endoproteolysis following wheat grain germination. Physiol. Plant. 1995, 95 (2), 253259.

(17) Kruger, J.; Preston, K. The nature and role of proteolytic enzymes during early germination. Cereal Res. Commun. 1976, 4 (2), 213-219.

(18) Corder, A. M.; Henry, R. J. Carbohydrate degrading enzymes in germinating wheat. Cereal Chem. 1989, 66 (5), 435-439.

(19) De Backer, E.; Gebruers, K.; Van den Ende, W.; Courtin, C. M.; Delcour, J. A. Post-translational processing of $\beta$-D-xylanases and changes in extractability of arabinoxylans during wheat germination. Plant Physiol. Biochem. 2010, 48 (2-3), 90-97.

(20) Meredith, P.; Jenkins, L. D. Communication to the editor. Amylase activity of sprout-damaged, malted and immature wheat. Cereal Chem. 1973, 50, 240-242.

(21) Kołodziejczyk, P.; Michniewicz, J. The changes of some enzymes activities during germination of rye kernels. Electron. J. Pol. Agric. Univ. 2004, 7 (1).

(22) Dornez, E.; Joye, I. J.; Gebruers, K.; Delcour, J. A.; Courtin, C. M. Wheat-kernel-associated endoxylanases consist of a majority of microbial and a minority of wheat endogenous endoxylanases. J. Agric. Food Chem. 2006, 54 (11), 4028-4034.

(23) AACC International. Approved methods of analysis, 11th ed.; AACC International: St. Paul, MN; http://methods.aaccnet.org/toc. aspx (accessed May 9, 2016).

(24) Struyf, N.; Verspreet, J.; Courtin, C. M. The effect of amylolytic activity and substrate availability on sugar release in non-yeasted dough. J. Cereal Sci. 2016, 69, 111-118.

(25) Brijs, K.; Trogh, I.; Jones, B. L.; Delcour, J. A. Proteolytic enzymes in germinating rye grains. Cereal Chem. 2002, 79 (3), 423428.

(26) Dupont, F. M.; Altenbach, S. B. Molecular and biochemical impacts of environmental factors on wheat grain development and protein synthesis. J. Cereal Sci. 2003, 38 (2), 133-146.

(27) Dedio, W.; Simmonds, D. H.; Hill, R. D.; Shealy, H. Distribution of $\alpha$-amylase in the triticale kernel during development. Can. J. Plant Sci. 1975, 55 (1), 29-36.

(28) Kruger, J. Changes in the amylases of hard red spring wheat during growth and maturation. Cereal Chem. 1972, 49 (4), 379-390.

(29) Mares, D. J. Temperature dependence of germinability of wheat (Triticum aestivum L.) grain in relation to pre-harvest sprouting. Aust. J. Agric. Res. 1984, 35, 115-128.

(30) Mares, D.; Mrva, K. Late-maturity $\alpha$-amylase: Low falling number in wheat in the absence of preharvest sprouting. J. Cereal Sci. 2008, 47 (1), 6-17.

(31) Kruger, J.; Tipples, K. H. Relationships between falling number, amylograph viscosity and $\alpha$-amylase activity in canadian wheat. Cereal Res. Commun. 1980, 8 (1), 97-105.

(32) Buonocore, V.; Petrucci, T.; Silano, V. Wheat protein inhibitors of $\alpha$-amylase. Phytochemistry 1977, 16, 811-820.
(33) Kruger, J. On the relationship between free and latent $\beta$ amylases in wheat. Cereal Chem. 1970, 47, 79-84.

(34) Cleemput, G.; Bleukx, W.; van Oort, M.; Hessing, M.; Delcour, $\mathrm{J}$. A. Evidence for the presence of arabinoxylan hydrolysing enzymes in European wheat flours. J. Cereal Sci. 1995, 22 (2), 139-145.

(35) Matthäus, K.; Dänicke, S.; Vahjen, W.; Simon, O.; Wang, J.; Valenta, H.; Meyer, K.; Strumpf, A.; Ziesenib, H.; Flachowsky, G. Progression of mycotoxin and nutrient concentrations in wheat after inoculation with Fusarium culmorum. Arch. Anim. Nutr. 2004, 58, 1935.

(36) Wrobel, R.; Jones, B. L. Appearance of endoproteolytic enzymes during the germination of barley. Plant Physiol. 1992, 100 (3), 15081516.

(37) Gys, W.; Courtin, C. M.; Delcour, J. A. Reduction of xylanase activity in flour by debranning retards syruping in refrigerated doughs. J. Cereal Sci. 2004, 39 (3), 371-377. 